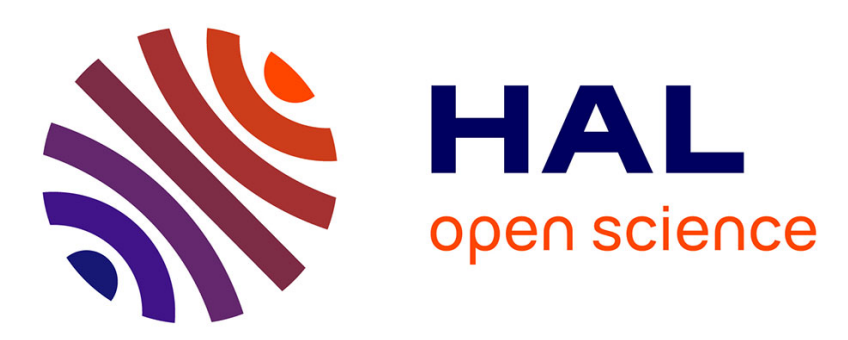

\title{
Monitoring based on time-frequency tracking of estimated harmonic series and modulation sidebands
}

\author{
Timothée Gerber, Nadine Martin, Corinne Mailhes
}

\section{To cite this version:}

Timothée Gerber, Nadine Martin, Corinne Mailhes. Monitoring based on time-frequency tracking of estimated harmonic series and modulation sidebands. CMMNO 2014 - 4th International Conference on Condition Monitoring of Machinery in Non-Stationary Operations, Dec 2014, Lyon, France. 10 p. hal-01044446v2

\author{
HAL Id: hal-01044446 \\ https://hal.science/hal-01044446v2
}

Submitted on 30 Jan 2015

HAL is a multi-disciplinary open access archive for the deposit and dissemination of scientific research documents, whether they are published or not. The documents may come from teaching and research institutions in France or abroad, or from public or private research centers.
L'archive ouverte pluridisciplinaire HAL, est destinée au dépôt et à la diffusion de documents scientifiques de niveau recherche, publiés ou non, émanant des établissements d'enseignement et de recherche français ou étrangers, des laboratoires publics ou privés. 


\title{
Monitoring Based on Time-Frequency Tracking of Estimated Harmonic Series and Modulation Sidebands
}

\author{
Timothée Gerber ${ }^{1}$, Nadine Martin ${ }^{2}$ and Corinne Mailhes ${ }^{3}$ \\ 1 Univ. Grenoble Alpes, GIPSA-lab, F-38000 Grenoble, France \\ timothee.gerber@gipsa-lab.grenoble-inp.fr \\ 2 CNRS, GIPSA-lab, F-38000 Grenoble, France \\ nadine.martin@gipsa-lab.grenoble-inp.fr \\ 3 IRIT/ENSEEIHT/TÉSA, University of Toulouse, France \\ corinne.mailhes@enseeiht.fr
}

\begin{abstract}
A condition monitoring system is a key element in a predictive maintenance strategy allowing to reduce the operating costs of the monitored system. However, the system-driven generation of health indicators requires the knowledge of the system kinematics and the configuration of thresholds which may induce lots of false alarms. In this paper, we propose a generic and data-driven method to automatically generate system health indicators without any a priori knowledge on the monitored system or the acquired signals. The proposed method is based on the automatic detection of spectral content characterising every acquired signal. Within these successive spectral contents, peaks, harmonics series and modulation sidebands are then tracked over time and grouped in time trajectories which will be used to generate the system health indicators.
\end{abstract}

Keywords: condition monitoring, tracking, surveillance, fault diagnosis, harmonics, sidebands, signal processing, wind turbines.

\section{Introduction}

The installation of a Condition Monitoring System (CMS) on a mechanical machine (e.g., on a wind turbine) aims to reduce the operating costs by applying a predictive maintenance strategy. The CMS is composed of sensors acquiring signals from which system health indicators are computed and monitored. System-driven computation of these indicators requires the monitored system kinematics and is done by averaging large or narrow spectral bands. The averaging and the need for predefined thresholds for fault detection may induce lots of false alarms while reducing the ability to detect a fault early.

To get precise health indicators whatever the system is, we propose a generic and data-driven monitoring strategy without any a priori knowledge on the system or the measured signals. The first step consists in analyzing and extracting 
the spectral content of each successive signal acquired by the CMS. This content is composed of single spectral peaks, or peaks grouped in more complex structures like harmonic series or modulation sidebands. Each spectral structure is characterized by several parameters, including for example the number of peaks, the characteristic frequencies and the energy [1].

The second step is a time-frequency tracking of the spectral structures through all available signals. It results in the creation of spectral structure trajectories from which the system health indicators will be derived. The time-frequency tracking method proposed in this paper is based on the McAulay \& Quatieri method [2] which has been originally designed for a single peak tracking in speech signals. We have adapted [2] in order to account not only for single spectral peak evolution but also for the evolution of more complex structures such as harmonic series or modulation sidebands. Moreover, the proposed method is made robust against the possible non-detection of spectral structures for some isolated signals among all the acquisitions thanks to a sleep state adapted from [3]. Finally, in every trajectory the temporal evolution of each spectral structure parameter can be monitored and used as precise system health indicators.

The paper is organised as follows. The identification of spectral peaks, harmonics series and modulation sidebands is presented in Section 2. Section 3 details the proposed time-frequency tracking method. In Section 4, the proposed method is validated on real-world signals acquired on a wind turbine test rig. Conclusions and perspectives are given in Section 5 .

\section{Identification of Spectral Peaks and Spectral Structures}

Different kinds of signals are recorded by a CMS: vibration, acoustic or electrical signals. In order to analyse a signal whatever its modality, we propose a generic and data-driven method to automatically identify the spectral peaks and the spectral structures like harmonic series or modulation sidebands. The different steps of the method illustrated in Fig. 1 are fully automatic and does not need any a priori knowledge or settings. Among a list of detected peaks, harmonic series and modulation sidebands are identified.

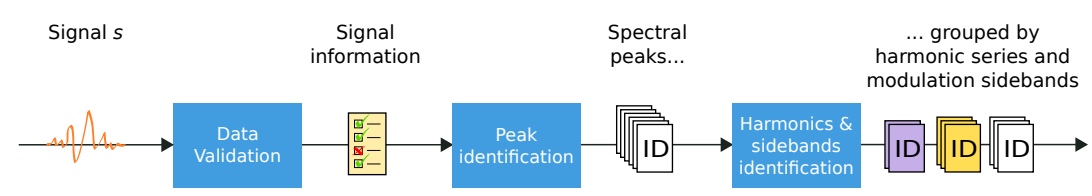

Fig. 1. The different steps of spectral content extraction: the data validation step assesses some global properties of the signal in order to ease the identification of spectral peaks which are finally grouped in harmonic series or modulation sidebands. 
Let us consider a sensor acquiring signals $s^{n}$ at time $t^{n}$, where $n$ denotes the signal index and $t^{n}$ is the time elapsed in operating hours since the beginning of the surveillance. Each signal is analysed independently by the following process. A preanalysis extracts fundamental information about the signal $s^{n}$ [4]. Then, a "multi-cycle" spectral analysis strategy [5] detects the peaks $\mathcal{P}_{i}^{n}$ present in the spectrum, with $i \in \llbracket 1, N_{\mathcal{P}}^{n} \rrbracket$ being the index of the peak and $N_{\mathcal{P}}^{n}$ being the total number of detected peaks. The list of peaks is sorted in ascending order of frequency.

Finally, the list of peaks is parsed to identify the harmonic series $\mathcal{H}_{j}^{n}$ and the modulation sidebands $\mathcal{M}_{k}^{n}$ present in the spectrum [1], where $j$ and $k$ are the index of the harmonic series and the modulation sideband respectively. These spectral structures are characterised by several parameters, including for example the number of peaks and the energy of the structure.

The identification of spectral peaks and structures in a sequence of signals $s^{n}$ is summarized in Fig. 2 by the discrete time-frequency map. Each detected peak is represented by a color and shape combination. Peaks with the same color and shape belong to the same spectral structure while the grey circles represent the peaks which does not belong to any structure. Further readings at $[5,1]$ for a detailed presentation of this steps.

\section{Time-Frequency Tracking of Peaks and Structures}

Time-frequency tracking is a problematic already present in the literature. Several methods are based on McAulay \& Quatieri one [2] which is simple and efficient but designed for peak tracking only. In [6], the peak tracking algorithm is based on a hidden Markov models and thus has a high computation complexity. Meanwhile, others methods $[7,8]$ are able to track spectral structures

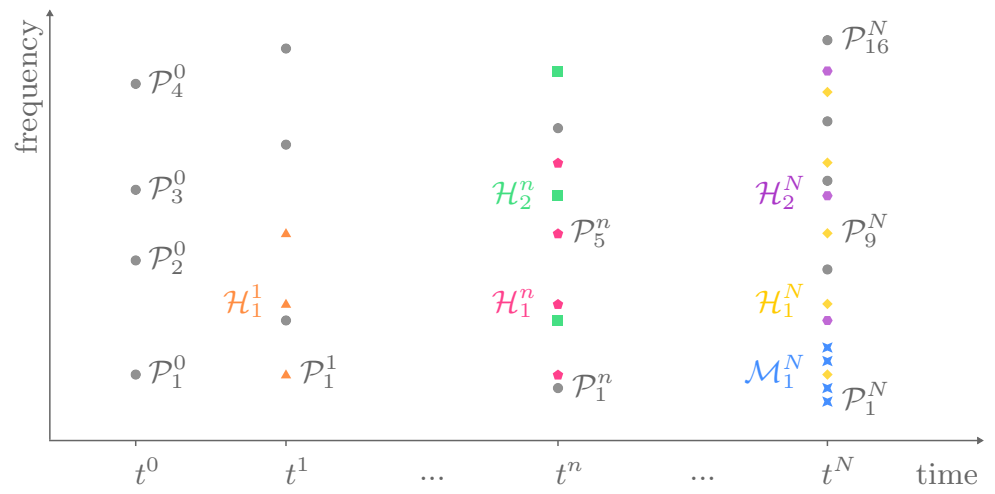

Fig. 2. A discrete time-frequency map illustrating the identification of peaks and structures for every signal acquired. Peaks which belong to the same structure share the same shape and colour. Grey circles represent the peaks which are not in a structure. 
like harmonic series. However, the number of structure tracked should be low or given a priori.

In our CMS context, the tracking method should be: (1) able to track both peaks and structures, (2) generic, thus necessitating no a priori information, and (3) able to deal with a large amount of signals, with thousands of peaks and hundreds of spectral structures. To our knowledge, none of the methods verifies all the criteria. Therefore, we propose to derive a method from the one of McAulay \& Quatieri which is the one with the lowest complexity. We adapted their method to track not only peaks but also spectral structures.

However, the CMS context is quite different from the audio signal context. The CMS acquires signals on a irregular temporal basis meaning that the state of the system could be completely different between two acquisitions. This is troublesome for the tracking operation. Therefore, the main hypothesis to use our proposed tracking method is that the signals are all acquired in a "constant machine state", where the operational parameters are constant during the acquisition and are the same for every acquisition. Angular resampling [9] may be performed as a pre-process to assess that the constant machine state hypothesis is verified.

\subsection{Tracking of Spectral Peaks}

The peak tracking is done sequentially and peak by peak, starting from the lowest frequencies. Suppose that the tracking is done up to time $t^{n}$ and up to peak $\mathcal{P}_{i-1}^{n}$. The trajectory $\mathcal{T}^{\mathcal{P}}$ of the next peak $\mathcal{P}_{i}^{n}$ will now be linked to a peak at time $t^{n+1}$ by the following 2 -step process.

Step 1: A search for candidate is made in the research interval $\Delta f$ around the frequency $f_{i}^{n}$. If there is no candidate, the trajectory of the peak $P_{i}^{n}$ dies. In the case of multiple candidates, the one with the nearest frequency to $f_{i}^{n}$ is elected as the best candidate.

Step 2: The best candidate from step 1 has to verify the backward compatibility condition. In other words, the frequency of the best candidate has to be closer to $f_{i}^{n}$ than to any other detected peak at time $t^{n}$. When the backward condition is met, the best candidate is added in the peak $\mathcal{P}_{i}^{n}$ trajectory. If there is no other candidate in the search interval, the trajectory of the peak $\mathcal{P}_{i}^{n}$ dies. Else, the association is made with the second best candidate in the search interval.

To sum up, the peak trajectories are constructed sequentially with

$$
\begin{gathered}
\mathcal{P}_{j}^{n+1} \in \mathcal{T}^{\mathcal{P}_{i}^{n}} \text { if } \underbrace{f_{i}^{n}-\frac{\Delta f}{2} \leq f_{j}^{n+1} \leq f_{i}^{n}-\frac{\Delta f}{2}}_{\text {search interval }} \\
\text { with } \underbrace{f_{j}^{n+1}=\underset{l=1, \ldots, N_{\mathcal{P}}^{n}}{\arg \min _{i}\left|f_{l}^{n}\right|} \text { and } \underbrace{\left|f_{i}^{n}-f_{j}^{n+1}\right|<\left|f_{i+1}^{n}-f_{j}^{n+1}\right|}_{\text {backward compatibility condition }}}_{\text {best candidate }} .
\end{gathered}
$$

In the original method, every peak is part of a trajectory, even if it is alone in its trajectory. We propose to start new trajectories only if at least two successive 
peaks will be included in it. Moreover, the original method is not robust against the possible non-detection of peaks, as the trajectory directly dies. We propose to introduce a sleep state as proposed in [3] to solve this problem. As a consequence, a trajectory can fall asleep and wake up. If the trajectory sleeps for a too long time (2 successive signals in our algorithm), the trajectory finally dies.

\subsection{Tracking of Harmonic Series}

The tracking of harmonic series is done by applying the strategy explained in 3.1 to the set of fundamental frequencies $f_{j}^{n}$ of all harmonic series $\mathcal{H}_{j}^{n}$. When two harmonic series are associated, the peaks inside the series are automatically tracked according to their harmonic rank. This structural approach is more ro-

bust than tracking each peak independently as the tracking is efficient even if some peaks are missing in the harmonic series. One of the missing peak can even be the peak representing the fundamental frequency.

\subsection{Tracking of Modulation Sidebands}

The modulation sidebands $\mathcal{M}_{k}^{n}$ are characterized by two parameters to track; the carrier frequency $f_{k}^{n}$ and the modulation frequency $\Delta f_{k}^{n}$. However, in the proposed method only the peaks belonging to at least one harmonic series could be consider as a potential carrier frequency. Therefore, the carrier frequency has already been tracked during the tracking of harmonic series (see 3.2).

It becomes then possible to track the modulation sidebands thanks to the strategy explained in 3.1 applied on the set of modulation frequencies $\Delta f_{k}^{n}$ present around each carrier frequency trajectory.

As for harmonic series, when modulation sidebands are tracked, the peaks inside the modulation are automatically tracked thanks to their modulation frequency. As a consequence, to track all the spectral content without redundancy, the tracking operations are done in the following order: (1) identification of the harmonic series trajectories $\mathcal{T}^{\mathcal{H}},(2)$ identification of the modulation sideband trajectories $\mathcal{T}^{\mathcal{M}}$, and (3) the identification of remaining peak trajectories $\mathcal{T}^{\mathcal{P}}$. The remaining peaks are the peaks which are not yet in any trajectory after harmonic and modulation tracking.

\subsection{Generation of the System Health Indicators}

The system health indicators are generated from the spectral structure trajectories. Each structure is characterised by several parameters, including for example the number of peaks and the energy [1]. The temporal evolution of these parameters for each trajectory are used as health indicators. Examples of these indicators are given in the next section of the paper. 


\section{Experiments}

\subsection{Description of the Wind Turbine Test Rig}

A test rig developed by the CETIM within the frame of the KAStrion project has been designed according to a wind turbine kinematics. Instead of the blades, a geared-motor of $10 \mathrm{~kW}$ generates the rotation of the main shaft (around 20 RPM). A multiplier with a ratio of 100:1 increases the rotational speed, allowing the generator to operate around 2000 RPM.

Among the several sensors installed on the test rig (accelerometers, thermocouples, torquemeters, tachometers, voltage and current probes), we will focus on the 3 accelerometers which take place on the main bearing and which are illustrated in Fig. 3. The $+x$ accelerometer is in the axial direction while the $+y$ and $-z$ are in radial directions.

The vibration acquisition is done while the test rig works under stationary conditions which remain the same for every acquisition. Therefore, the hypothesis of constant machine state is met. Each vibration signal lasts 150 seconds and is sampled at $39062.5 \mathrm{~Hz}$.

One of the rig tasks consists in damaging the main bearing thanks to a loading unit and non-stationary working conditions which have been programmed to simulate real wind speed profiles. The results of the proposed method on the degradation of two different main bearings is presented in 4.2 and 4.3.

\subsection{Medium Degradation of the Main Bearing}

For each accelerometer, 12 vibration signals were recorded during the main bearing medium degradation. In each signal, an average of 19400 peaks were detected and grouped in 750 harmonic series and 21500 modulation sidebands. If these

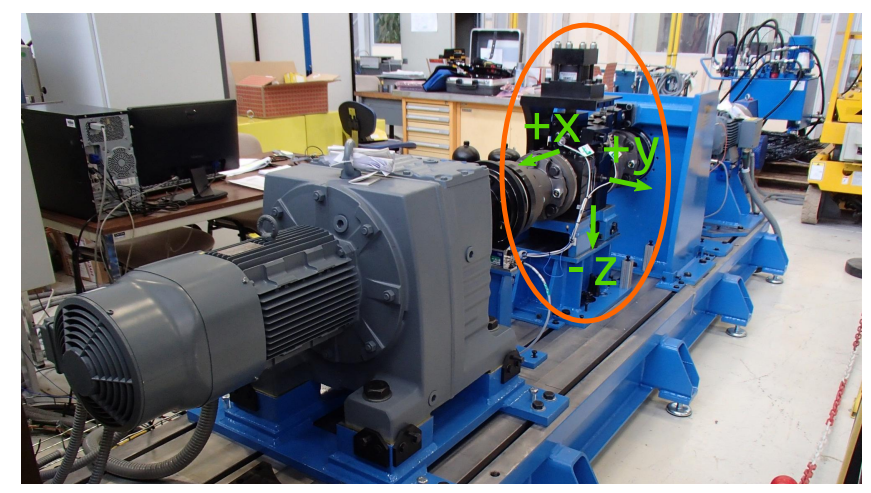

Fig. 3. A picture of the wind turbine test rig. The 3 accelerometers are represented by green arrows. The main bearing and its loading unit is circled in orange. 
numbers seem large, one has to remember that the identification methods operate over all the signal frequency band without any a priori.

Let us first consider the accelerometer in the $-z$ direction. After the tracking operations, 1084 harmonic series trajectories and 564 modulation sideband trajectories are identified. Among the remaining peaks, 9327 trajectories are created.

One of the trajectory is particularly interesting. It is a harmonic series trajectory which energy suddenly and rapidly increases from approximatively 0.011 to $1.6 \mathrm{\mu m}^{2} . \mathrm{s}^{-2}$ after 203 operating hours (see Fig. 4). The fundamental frequency of this harmonic series is $2.72 \mathrm{~Hz}$ and corresponds to the Ball Pass Frequency of the Outer ring (BPFO) of the main bearing.

The wear test is stopped after 214 operating hours although the rig is still able to operate. The main bearing is dismantled for visual inspection; 3 small flaking were present on the outer ring. This confirms the relevancy of the automatically generated indicator.

The results of the other accelerometers $(+x,+y)$ will not be presented here as they show high similarity to the result of accelerometer $-z$.

\subsection{Full Degradation of the Main Bearing}

For this second wear test, another kind of main bearing is used with a different kinematics. Let us consider the $+y$ accelerometer. 17 vibration signals were recorded, containing an average of 9000 peaks, 600 harmonic series and 12000 modulation sidebands. After the tracking operations, we get 828 harmonic series trajectories and 9373 modulation sideband trajectories. 12406 peak trajectories are created among the remaining peaks.

Fig. 5 shows one harmonic series trajectory deserving a special attention. On the top of the figure, peaks are symbolised by small grey circles on the discrete time-frequency map. The time localization of the peaks correspond to the signal acquisition timestamp. The zoom in the top left corner shows the high peak density. Among these detected peaks, a particular peak at $3.45 \mathrm{~Hz}$ is tracked from 44 to 189 operating hours and is represented by bigger and blue circles. About 129 hours, this peak trajectory evolves to become a harmonic

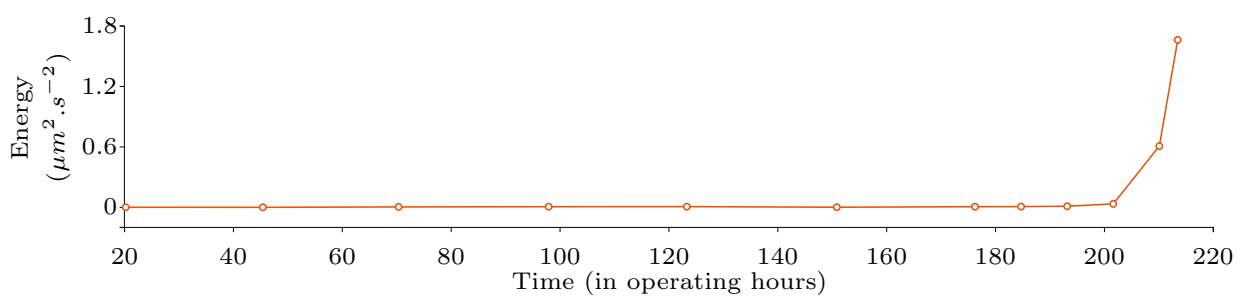

Fig. 4. Medium degradation of the main bearing. The energy in the inner ring harmonic series starts increasing around 200 operating hours. After 214 hours, the test is stopped and the bearing is dismantled for inspection. 
series trajectory with more and more peaks (see the increasing number of blue circles). Also, the energy of this trajectory increases and is represented by the orange plot in the middle of Fig. 5. The last plot in the bottom of Fig. 5 shows that the frequency of the trajectory is slightly decreasing.

Moreover, a $0.33 \mathrm{~Hz}$ modulation sidebands started to appear after 134 operating hours around the $3.45 \mathrm{~Hz}$ carrier frequency. As shown in Fig. 6, the number of sidebands increases accordingly to the severity of the fault.

The $3.45 \mathrm{~Hz}$ corresponds to the Ball Pass Frequency of the Inner ring (BPFI). The apparition of harmonics and sidebands at 129 and 134 operating hours respectively are an early warning of a fault birth. The fault severity is then characterized by the increase in both energy and number of sidebands. The slightly decreasing frequency is explained by the fact that the inner ring wear generates slipping.

A second harmonic series trajectory also deserves some attention. Its fundamental frequency of $2.54 \mathrm{~Hz}$ corresponds to the BPFO. As shown in Fig. 7, its
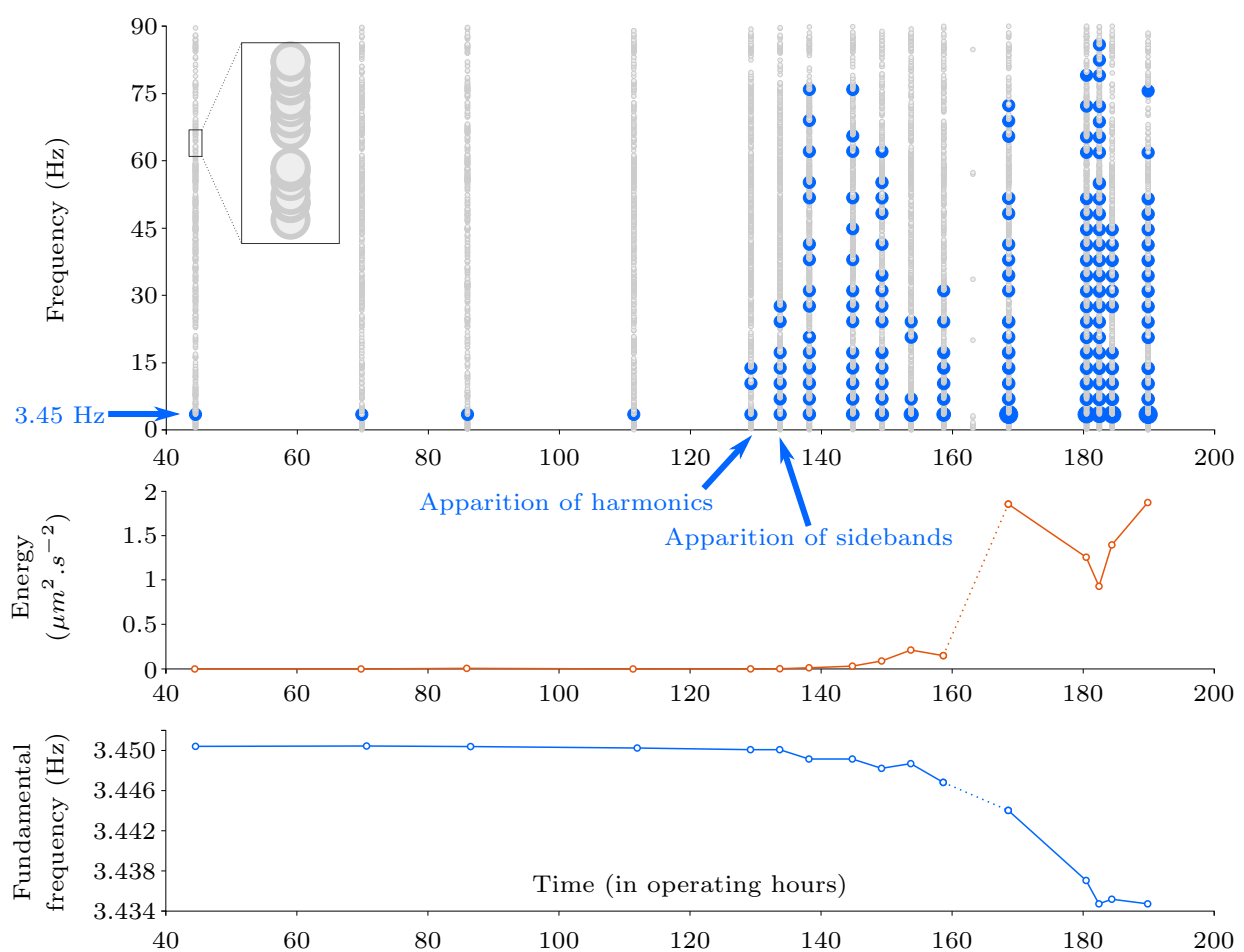

Fig. 5. Evolution of the main bearing fault, seen by the $+y$ accelerometer. On the top, all detected peaks are represented by small grey circles on the time-frequency map. Blue and bigger circles belongs to the same harmonic series trajectory linked to the inner ring fault. Below, two trends of the harmonic series are plotted: the energy increases while the fundamental frequency is slightly decreasing. 
energy starts increasing from 144 operating hours, that is 15 operating hours after the early warning of the BPFI fault. It means that the fault is spreading in the main bearing and its severity is increasing.

On the top of Fig. 5, it is possible to see on the time-frequency map that around 163 hours few peaks were detected in the signal. The spectral analysis was not able to identify the peaks and structures for this particular signal. Indeed, other tests [4] were applied and concluded on the fact that this signal was highly non-stationary due to a high impulse in the vibration signal. Nervertheless, the tracking of harmonic series and modulation sidebands was not stopped as it is shown in Fig. 5, 6 and 7. In fact, the sleep state allowed the tracking to continue. The sleep state is represented by a dotted line on the different curves.

The combination of the four automatically generated indicators (the energy and the frequency of the harmonic series at $3.45 \mathrm{~Hz}$, the number of sidebands around the carrier frequency $3.45 \mathrm{~Hz}$ and the energy of the harmonic series at $2.54 \mathrm{~Hz}$ ) mirrors out the failure and confirms the value the proposed data-driven method. These four indicators are part of a long list of automatically generated indicators. In order to make a full automatic system health diagnosis, further work will focus on identifying automatically the most interesting indicators.

\section{Conclusions}

We proposed in this paper a complete generic and data-driven method to automatically generate system health indicators. The two steps of the proposed

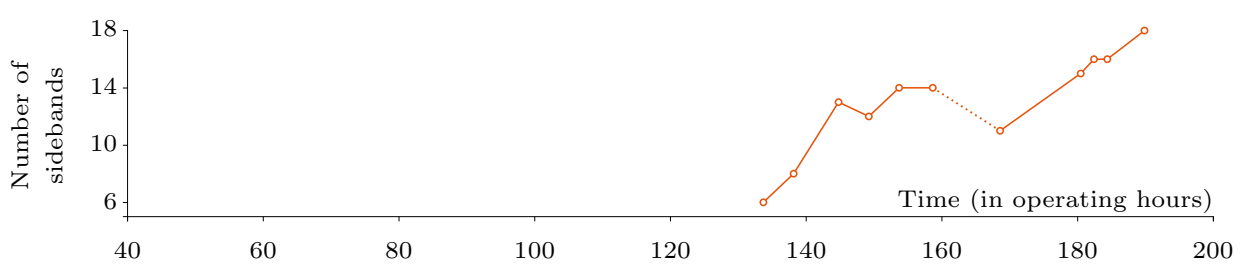

Fig. 6. Evolution of the number of modulation sidebands around the slowly evolving carrier frequency starting at $3.45 \mathrm{~Hz}$. The modulation frequency is equal to $0.333 \mathrm{~Hz}$.

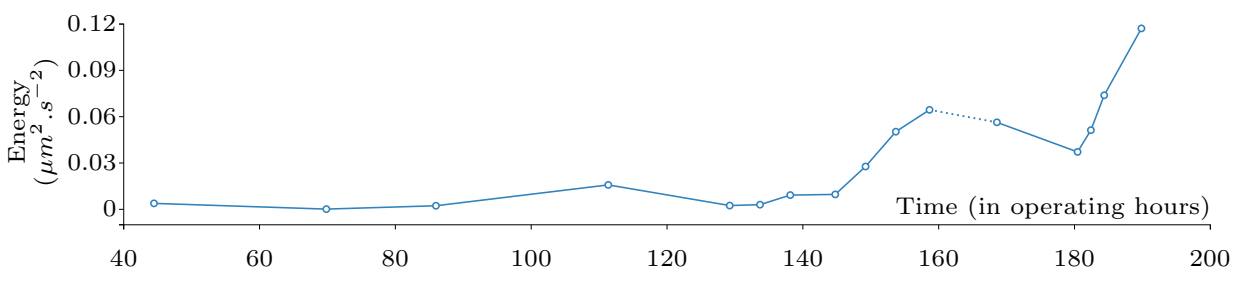

Fig. 7. Propagation of the fault: the energy of the outer ring harmonic series is also increasing after 145 operating hours. 
method are (1) the identification of the spectral structures present in each signal spectrum, and (2) the tracking of these detected spectral structures through all the available signals. The trajectories constructed during the tracking operations are finally used to derive the system health indicators from the structure parameters.

The proposed approach is validated on real-world signals, recorded on a wind turbine test rig. Two different main bearings have been damaged. In both cases, the system health indicators automatically generated could detect the fault in its early stage. The severity of the faults is characterised by the time evolution of the health indicators.

The number of system health indicators generated is large. Therefore, future work will focus on sorting and classifying these indicators to make a fully automatic tool for system diagnosis.

Acknowledgements. The KAStrion project has been supported by KIC InnoEnergy, a company supported by the European Institute of Innovation and Technology (EIT), and has the mission of delivering commercial products and services, new businesses, innovators and entrepreneurs in the field of sustainable energy through the integration of higher education, research, entrepreneurs and business companies.

We would also like to thank the CETIM, partner of the project, for providing the signals coming from the wind turbine test rig.

\section{References}

1. T. Gerber, N. Martin, and C. Mailhes, "Identification of harmonics and sidebands in a finite set of spectral components," in CM $\mathscr{G}$ MFPT 2013, (Kraków), 2013.

2. R. McAulay and T. Quatieri, "Speech analysis/Synthesis based on a sinusoidal representation," IEEE Transactions on Acoustics, Speech, and Signal Processing, vol. 34, pp. 744-754, Aug. 1986.

3. X. Serra, "Musical sound modeling with sinusoids plus noise," Musical signal processing, pp. 1-25, 1997.

4. N. Martin and C. Mailhes, "A non-stationary index resulting from time and frequency domains," in CM \& MFTP 2009, 2009.

5. C. Mailhes, N. Martin, K. Sahli, and G. Lejeune, "Condition monitoring using automatic spectral analysis," in Structural Health Monitoring, (Spain), 2006.

6. P. Depalle, G. Garcia, and X. Rodet, "Tracking of partials for additive sound synthesis using hidden Markov models," Acoustics Speech and Signal Processing, 1993.

7. R. C. Maher and J. W. Beauchamp, "Fundamental frequency estimation of musical signals using a two-way mismatch procedure," The Journal of the Acoustical Society of America, vol. 95, no. April 1994, pp. 2254-2263, 1994.

8. Z. Duan, J. Han, and B. Pardo, "Multi-pitch Streaming of Harmonic Sound Mixtures," IEEE/ACM Transactions on Audio, Speech, and Language Processing, vol. 22 , pp. 138-150, Jan. 2014.

9. M. Firla, Z.-Y. Li, N. Martin, and T. Barszcz, "Full-band Demodulation in Automatic Application for Fault Detection - a Wind Turbine Test Rig Case Study," in Condition Monitoring of Machinery in Non-Stationary Operations, 2014. 